\title{
Quality Assessment of High-Resolution Images with Small Distortions after Compression
}

\author{
Tomas PETKUS ${ }^{1}$, Jevgenij TICHONOV ${ }^{2}$, Ernestas FILATOVAS ${ }^{3}$, \\ Vytautas JAKŠTYS ${ }^{2}$ \\ ${ }^{1}$ Lithuanian University of Educational Sciences, Faculty of Science and Technology, Studentų str. \\ 39, LT-08106 Vilnius, Lithuania \\ ${ }^{2}$ Vilnius University, Institute of Mathematics and Informatics, Akademijos str. 4 \\ LT-08663 Vilnius, Lithuania \\ ${ }^{3}$ Vilnius Gediminas Technical University, Faculty of Fundamental Sciences, Sauletekio av.11-615, \\ LT-10223, Vilnius, Lithuania \\ tomas.petkus@leu.lt, jevgenij.tichonov@mii.vu.lt, \\ ernestas.filatovas@vgtu.lt, vytautas.jakstys@mii.vu.lt
}

\begin{abstract}
Image quality assessment still remains a highly relevant problem, and objective quality assessment methods are being actively developed. The methods, based on the Structural Similarity index method, have become very popular during the last decade. However, their sensitivity has been investigated using only small images and only in the cases of obvious image distortions. In this paper, we have investigated a quality assessment of high-resolution images with low distortions after compression using the Structural Similarity index method. The specific cases, related to the usage of this method for high-resolution images, are analyzed, and the problems that occur when using the method are identified. Experimental investigations have shown that image downsampling is necessary when the image quality is assessed by the Structural Similarity index method. Moreover, a sensitive algorithm suitable for the comparison of the quality of highresolution images with small distortions is proposed and investigated in the paper.
\end{abstract}

Keywords. Image processing, image quality, high-resolution imaging, SSIM index method

\section{Introduction}

As image processing has become one of the essential parts in the digital information industry, great efforts are directed to the development of image quality assessment methods during the last 20 years. Image quality assessment methods play an important role in a wide range of applications, and they are based on a comparison of the original and distorted (after compression) images, which is represented by the numeric expression. However, a subjective people's impression remains the most important indicator for image quality comparison. The point of the subjective method, called subjective Mean Opinion Score (MOS), is based on the average of the opinion scores, provided by a group of experts on the quality of the compressed image (Ponomarenko et al., 2009a). The main disadvantage of the method is that it is expensive and time consuming (Wang et al., 2004a). Another essential disadvantage of the method is that the 
human eye fails to notice small artefacts while assessing high-resolution images. For example, the same image could be modified by many variants of the JPEG algorithm, but the human visual perception would not notice any changes in the distorted images. For these reasons, objective methods are often used for image quality assessment (Wang and Bovik, 2009). A comprehensive review of such methods is given in (Thung and Raveendran, 2009), (George and Prabavathy, 2014), (Joy and Sarma, 2014).

The most wide-used method for image quality assessment is Peak Signal-to-Noise Ratio (PSNR) (Hore and Ziou, 2010), (Salomon, 2013). The basis of this method is the calculation of the Mean Square Error (MSE). But it should be noted that a high PSNR value not always guarantees a good image quality (Wang and Bovik, 2009), (Hore and Ziou, 2010). Another popular method for image quality assessment is a Structural Similarity (SSIM) index method (Wang et al., 2004a). It is more complex, but accurate, therefore this method has become of great interest during the last decade. In order to evaluate the objective metrics correspondence to human impression, the image databases are created. A comprehensive survey of such databases is presented in (Winkler, 2012) (Chandler, 2013). The image databases have reference images, and the images, distorted by various noises, as well as MOS values estimating expert opinions on each image (Ponomarenko et al., 2009b). For example, the TID2013 database (Ponomarenko et al., 2013) contains 1700 images, where 68 distorted images (four levels for each of seventeen types of distortions) have been obtained for each reference image. In total, 985 estimations were performed in five countries (Ukraine, Finland, France, USA, and Italy). Researches have shown that, in many cases of distorted images, the SSIM index-based methods provide very good values correlated with the values of the Human Visual System (HVS) (Ponomarenko et al., 2009b), (Ponomarenko et al., 2013). The HVS value is obtained by determining the correlation value between MOS and the "ideal" MOS when the number of experiments approaches infinity.

Nowadays huge sets of high-resolution digital images are used in medicine, astronautics, social networks, etc. Such images must be automatically compressed using JPEG (Wallace, 1992), JPEG2000 (Skodras et al., 2001) or other compression methods in order to reduce the amount of occupied memory while maintaining the same resolution. Moreover, it is often important to keep the sufficient high quality of images, when distortions are hardly noticeable. However, the state-of-art methods, such as the Structural Similarity index method used for image quality assessment, are suitable only in the case of obvious distortions. Moreover, the SSIM index method and its extensions were successfully tested only on small images with big distortions (Ponomarenko et al., 2013). As it is demonstrated in the paper hereafter, the quality assessment problems arise, where large and high-resolution images with hardly noticeable changes are investigated. Therefore, methods for evaluating high-resolution images with even small distortions should be developed.

In this paper, we identify and highlight weaknesses of the existing SSIM index method-based algorithms. The specific cases, related to the usage of the SSIM index method, are analyzed. It is identified here that the original SSIM index method is not suitable for quality assessment of high-resolution images with low distortions. It is shown that downsampling is necessary when the high-resolution image quality is assessed by the SSIM index method. We propose a modified algorithm on the basis of the SSIM index method that is suitable for the comparison of high-resolution images.

The remainder of this paper is organized as follows. The concepts of the SSIM index method and its extensions, as well as their application areas, are discussed in Section 2. In Section 3, we analyze the suitability of the original SSIM index method for quality 
assessment of high-resolution images with small distortions and identify the occurring problems. The results of the experimental investigations on image data downsamling before the usage of the SSIM index method are presented in Section 4. The algorithm suitable for quality assessment of high-resolution images with small distortions is also proposed and experimentally investigated here. Finally, conclusions are drawn in Section 5.

\section{Concepts of structural similarity}

The SSIM index method and its extensions are widely used for image and video processing (Russ, 2011), (Fernando et al., 2013), and for the related areas such as image classification (Gao et al., 2011), image restoration and fusion (Piella and Heijmans, 2003), (Rehman et al., 2012), distortion optimization (Huang et al., 2010), watermarking and biometrics (Wang and Bovik, 2009).

The SSIM index method assesses the visual impact of three characteristics of an image: luminance, contrast, and structure. The overall SIMM index value is a multiplicative combination of these characteristics. With two local image patches given (a patch of the original image $x$ and a patch of the distorted image $y$ ), the local SSIM index value is defined as (Wang et al., 2004a):

$$
S(x, y)=[l(x, y)]^{\alpha} \times[c(x, y)]^{\beta} \times[s(x, y)]^{\gamma},
$$

where

$$
\begin{aligned}
& l(x, y)=\frac{2 \mu_{x} \mu_{y}+C_{1}}{\mu_{x}^{2}+\mu_{y}^{2}+C_{1}}, \\
& c(x, y)=\frac{2 \sigma_{x} \sigma_{y}+C_{2}}{\sigma_{x}^{2}+\sigma_{y}^{2}+C_{2}}, \\
& s(x, y)=\frac{\sigma_{x y}+C_{3}}{\sigma_{x} \sigma_{y}+C_{3}} .
\end{aligned}
$$

Here $l(x, y), c(x, y)$, and $s(x, y)$ are the local luminance, the local contrast, and the local structure, respectively; $\mu_{x}, \mu_{y}, \sigma_{x}, \sigma_{y}$, and $\sigma_{x y}$ are the local means, the standard deviations, and the cross-correlation for the local image patches $x$ and $y$, respectively. $\alpha>0, \beta>0$ and $\gamma>0$ are parameters used for adjustment of a relative importance of the three components. The constants $C_{1}$ and $C_{2}$ are used to avoid instability when the means and deviations are close to zero. The values, obtained by formula (1), compose the so-called SSIM index map. The SSIM index value of the whole image is obtained by averaging the local SSIM index values, calculated by using a sliding window of the size $8 \times 8$ pixels. The SSIM index method measures the similarity between the two images and the similarity value lies between -1 and 1 . If $\alpha=\beta=\gamma=1$ and $C_{3}=C_{2} / 2$, the SSIM index value is simplified up to: 


$$
S(x, y)=\frac{\left(2 \mu_{x} \mu_{y}+C_{1}\right)\left(2 \sigma_{x y}+C_{2}\right)}{\left(\mu_{x}^{2}+\mu_{y}^{2}+C_{1}\right)\left(\sigma_{x}^{2}+\sigma_{y}^{2}+C_{1}\right)} .
$$

In spite of the fact that the mathematical model of the SSIM index method is rather complex, it is considered that the resulting indicator sufficiently reflects the human visual perception features. Mathematical properties of the method are comprehensively discussed in (Brunet et al., 2012).

It should be noted that the distance between an image and a human can affect his/her perception of image quality. For this reason, a Multi-Scale SSIM (MS-SSIM) index was developed and formulated as follows (Wang et al., 2004b):

$$
S(x, y)=[l(x, y)]^{\alpha_{P}} \times \prod_{i=1}^{P}[c(x, y)]^{\beta_{i}}[s(x, y)]^{\gamma_{i}} .
$$

Here the contrast and structure are calculated at each scale $i$, and the luminance is computed only at scale $P$. The overall quality evaluation is obtained by combining the measurements over different $P$ scales.

It is obvious that the MS-SSIM index has a higher correlation with HVS for relatively small images, where the image quality for a human depends on the distance from an eye to the image (scale). However, in the case of large images with small distortions, presented in a high resolution, there is no such dependence. Further, several image quality assessment methods have been developed on the basis of the SSIM index method: a HWSSIM method (Ji et al., 2008), where a discrete Haar wavelet transform is used, a DWT-SSIM method (Yang et al., 2008), in which a discrete wavelet transform is applied, a PSSIM ${ }^{\mathrm{c}}$ method (Yang et al., 2008), where the SSIM index values of local image regions are adjusted by perceptual weights defined from the contrasts of the regions.

Despite the variety of the SSIM-based methods, in this paper, we focus on the investigation of the original SSIM index method (Wang et al., 2004a), which is the basis of the others.

\section{Suitability analysis of the SSIM index method}

In this section, we analyze the suitability of the original SSIM index method for quality assessment of high-resolution images with small distortions and identify the occurring problems.

In the experimental investigations, a set of 30 different high-resolution images $(3882 \times 2608$ pixels, $28.9 \mathrm{MB})$ was used for calculating the image similarity and SSIM index values. The selected images were modified by Spencer Kimball and Peter Mattis JPEG storage procedure which had been implemented in GIMP 2.6.11 (WEB, a). The default settings were kept the same, only the Quality Factor (QF) was changed. The QF is the main parameter influencing the image quality after JPEG compression, which determines the compression ratio. This parameter is an integer number between 0 and 100 , used to parameterize a quantization matrix. The greater this number is, the less information is lost. 
The compression algorithm was implemented by the Independent JPEG Group (IJG) (WEB, b). In the experimental investigation, three different compression quality factor values $(85,70$, and 50) are used. The SSIM index values are calculated for each image from a set of 30 different images, using the algorithm developed by Zhou Wang and implemented in MATLAB (WEB, c) (we call it Algorithm 1). The obtained SSIM index values are averaged are presented in Table 1 .

Table 1. Average of the SSIM index values obtained by Algorithm 1

\begin{tabular}{|c|c|c|}
\hline Compression level & \multicolumn{1}{|l|}{ SSIM } & File size \\
\hline Original & 1.0000 & $29.8 \mathrm{MB}$ \\
\hline JPEG 85 & 0.9999 & $897 \mathrm{~KB}$ \\
\hline JPEG 70 & 0.9998 & $527 \mathrm{~KB}$ \\
\hline JPEG 50 & 0.9985 & $355 \mathrm{~KB}$ \\
\hline
\end{tabular}

We can see that file sizes of the images are different, however, the averaged SSIM index values are very close to 1 . This is due to the fact that the mathematical model of the SSIM index method and the resulting index values reflect the human visual perception of distortions very well. In this case, a human eye would not easily fix the obvious differences between high-resolution images, therefore the SSIM index values are very high. An illustration of this fact is presented in Fig. 1. Only after zooming one of the fragments of the examined image, we can see that the differences between the original and distorted images are obvious.

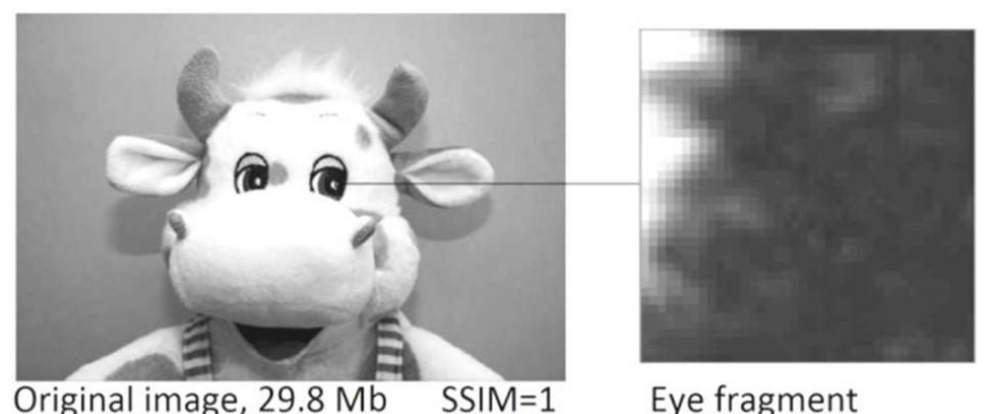

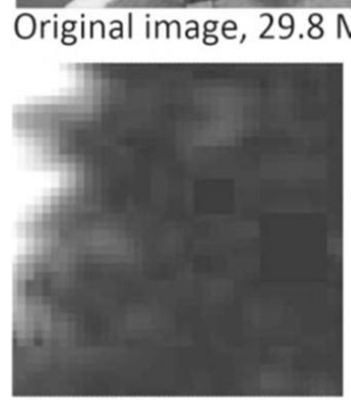

a) JPEG $85 \quad 888 \mathrm{~KB}$ $\mathrm{SSIM}=0.9998$

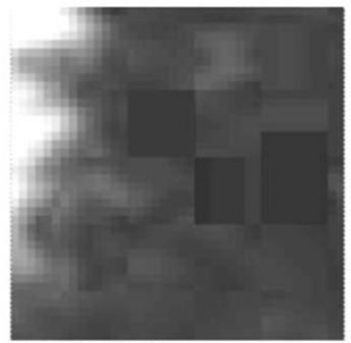

b) JPEG $70 \quad 512 \mathrm{~KB}$ $\mathrm{SSIM}=0.9998$

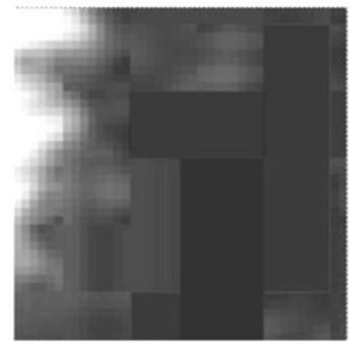

c) JPEG $50333 \mathrm{~KB}$ $\mathrm{SSIM}=0.9982$

Fig. 1. Fragments of an image changed by JPEG algorithms, using various values of the image quality level (SSIM index is computed by Algorithm 1). 
Here, in the cases a) and b), the SSIM index values are the same, however, the quality of the images is very different (when the values are expressed by four digits after the comma). Moreover, as we see in the case c), the SSIM index value has changed slightly, after a significant change in the image quality. The problem is that the SSIM index values look similar for the human and do not reflect the distortion as much as it should be while exploring the JPEG algorithm variants. This problem is particularly relevant if large groups of images must be processed and quality assessment must be automatic. Although a human could not notice some distortions in the compressed images, however, these images must be of a high quality for further processing, e.g. for some pattern recognition, for pressing the images. Moreover, in the case of medical images, they must be saved of a high quality and high resolution for accurate diagnosis.

\section{Image data downsampling before calculating the SSIM index values}

Image reduction (downsampling) is typically used before the assessment of image quality by the SSIM index method (Wang et al., 2004a). The image reduction coefficient $F$ is calculated by the following empirical formula:

$$
F=\max (1, \operatorname{round}(\min (M, N) / f)) \text {, }
$$

where $N$ is the width and $M$ is the length of the image; $f$ is the dimension size of the downsampled image, which is suggested to be 256 pixels [2]. For example, for the image with dimensions of $1536 \times 1536, F$ value is 6 , thus it should be reduced by coefficient 6 . The reduction of an image fragment of size $F \times F$ is processed by the following formula:

$$
g\left(F^{2}\right)=\sum_{i=1}^{F} \sum_{j=1}^{F} \frac{i m g[i, j]}{F^{2}},
$$

where $i m g[i, j]$ is the value of every pixel. The calculation of this formula is repeated from 1 to $M$ and from 1 to $N$ by the step $F$. This image reduction procedure is used in Algorithm 1 and illustrated in Fig. 2.

The case, where SSIM index value is computed without downsampling, is called Algorithm 2. In Table2, the comparison of Algorithm 1 and Algorithm 2 is presented when assessing the quality of compressed images by the SSIM index method. We see that when using Algorithm 2, the obtained SSIM index values differ more, i.e. they better reflect changes (and distortions) in the images. We conclude that Algorithm 2 is more suitable for quality assessment of images with small distortions, however, it has some disadvantages.

Firstly, Algorithm 2 is time consuming, due to the fact that large amounts of data must be processed. For evidence, the calculation speed of the SSIM index values has been investigated and performance of the algorithms has been compared. The experimental investigation is carried out in the MATLAB R2012b system, using a computer with a Pentium DualCore T4200 processor and 4GB of RAM. A selected set of 30 different images of sizes $3882 \times 2608$ and $1941 \times 1304$ was used. Experiments are 
performed for 10 times with each image using Algorithm 1 and Algorithm 2. It has been obtained that Algorithm 1 is approximately two times faster, when the size of the images is $1941 \times 1304$ pixels $(F=5)$ and even seven times faster, when the size of images is $3882 \times 2608$ pixels $(F=10)$, comparing with Algorithm 2 .

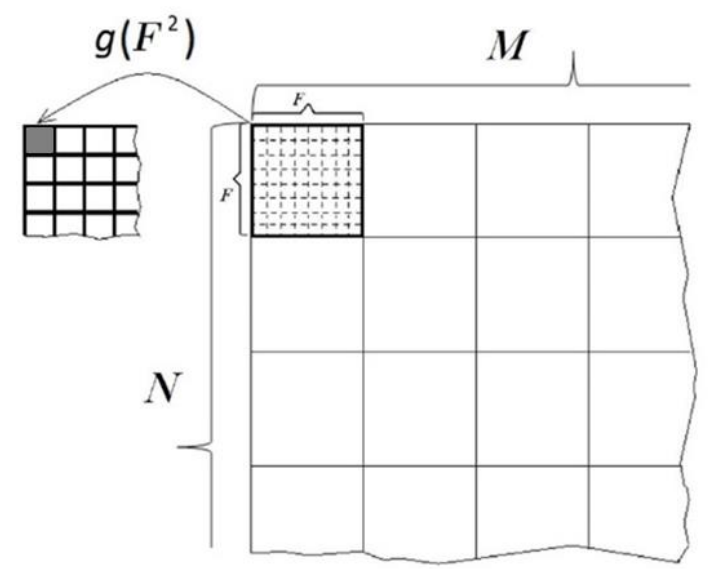

Fig. 2. The image downsampling typically used in the SSIM index method.

Table 2. The SSIM index values obtained by algorithm 1 and algorithm 2

\begin{tabular}{|l|c|c|}
\hline Compression level & $\begin{array}{c}\text { SSIM } \\
\text { (Algorithm 1) }\end{array}$ & $\begin{array}{c}\text { SSIM } \\
\text { (Algorithm 2) }\end{array}$ \\
\hline Original & 1.0000 & 1.0000 \\
\hline JPEG 85 & 0.9999 & 0.9765 \\
\hline JPEG 70 & 0.9998 & 0.9661 \\
\hline JPEG 50 & 0.9985 & 0.9558 \\
\hline
\end{tabular}

Firstly, Algorithm 2 is time consuming, due to the fact that large amounts of data must be processed. For evidence, the calculation speed of the SSIM index values has been investigated and performance of the algorithms has been compared. The experimental investigation is carried out in the MATLAB R2012b system, using a computer with a Pentium DualCore T4200 processor and 4GB of RAM. A selected set of 30 different images of sizes $3882 \times 2608$ and $1941 \times 1304$ was used. Experiments are performed for 10 times with each image using Algorithm 1 and Algorithm 2. It has been obtained that Algorithm 1 is approximately two times faster, when the size of the images is $1941 \times 1304$ pixels $(F=5)$ and even seven times faster, when the size of images is $3882 \times 2608$ pixels $(F=10)$, comparing with Algorithm 2 .

A second more significant disadvantage of Algorithm 2 is that the SSIM index values are highly dependent on the size of images, especially when the structures of the distorted images are changed. The analysis of the dependence of the SSIM index values on the image size using the Algorithm 2 has been made. The steps of the analysis are as follows: 
- The original image of $512 \times 512$ pixels in size is distorted by one of the most popular types of distortions: brightening, increase of the contrast, impulsive noise, blurring, and compression by the JPEG algorithm.

- The edges of the original image are increased by 4 times up to the image size of $2048 \times 2048$ pixels without using any interpolations and quality improvements. For example, when increasing an image from the size $M \times N$ twice to the size $2 M \times 2 N$, each pixel is quadrupled, but the structure and proportions of the image are kept.

- The distorted images are also increased by 4 times.

Table 3. Dependence of the SSIM index values on the type of distortion

\begin{tabular}{|l|c|c|}
\hline Type of distortion & $\begin{array}{c}\text { Image size } \\
\mathbf{5 1 2 \times 5 1 2}\end{array}$ & $\begin{array}{c}\text { Image size } \\
\mathbf{2 0 4 8} \times \mathbf{2 0 4 8}\end{array}$ \\
\hline Original & 1 & 1 \\
\hline Brightening & 0.937 & 0.922 \\
\hline Contrast increase & 0.923 & 0.911 \\
\hline Impulsive noise & 0.723 & 0.902 \\
\hline Blurring & 0.731 & 0.767 \\
\hline JPEG algorithm & 0.681 & 0.729 \\
\hline
\end{tabular}

These steps with various distortions are performed for the set of 30 different images, the averages of the SSIM index values for the images of equal sizes are calculated and presented in TABLE 3. We can see that the SSIM index values differ depending on the type of distortion. The largest differences between the SSIM index value of the distorted images and the value of the original images are obtained if the images are distorted by the impulse noise.

For illustration, one original image, the distorted images, and the SSIM index values for images of different sizes are presented in Fig. 3: (a) - original image, (b) brightening, (c) - increase of the contrast, (d) - impulsive noise, (e) - blurring, and (f) compression by the JPEG algorithm. The different SSIM index values are obtained for different size of images distorted by the same type of distortion.

The image distorted by increasing the contrast and the SSIM maps of the distorted images of different sizes are presented in Fig. 4. It can be noticed that, if the image is processed by increasing the contrast, the SSIM maps are almost similar. In this case, the image size does not influence the values of the SSIM index. In Fig. 5, the image distorted by impulse noise and the SSIM maps are presented. We see that the obtained SSIM maps are quite different when the image is processed by impulsive noise (the structure is changed). Thus, the image size influences the SSIM maps and the SSIM index values thereby. It is concluded that when assessing large high-resolution images, the algorithm without downsampling cannot be used for computing the SSIM index values, due to the fact that the obtained values would be unreliable.

It is obvious that image downsampling must be processed, but its implementation in Algorithm 1 by formula (8) is not suitable for assessment of high-resolution images with small distortions. 

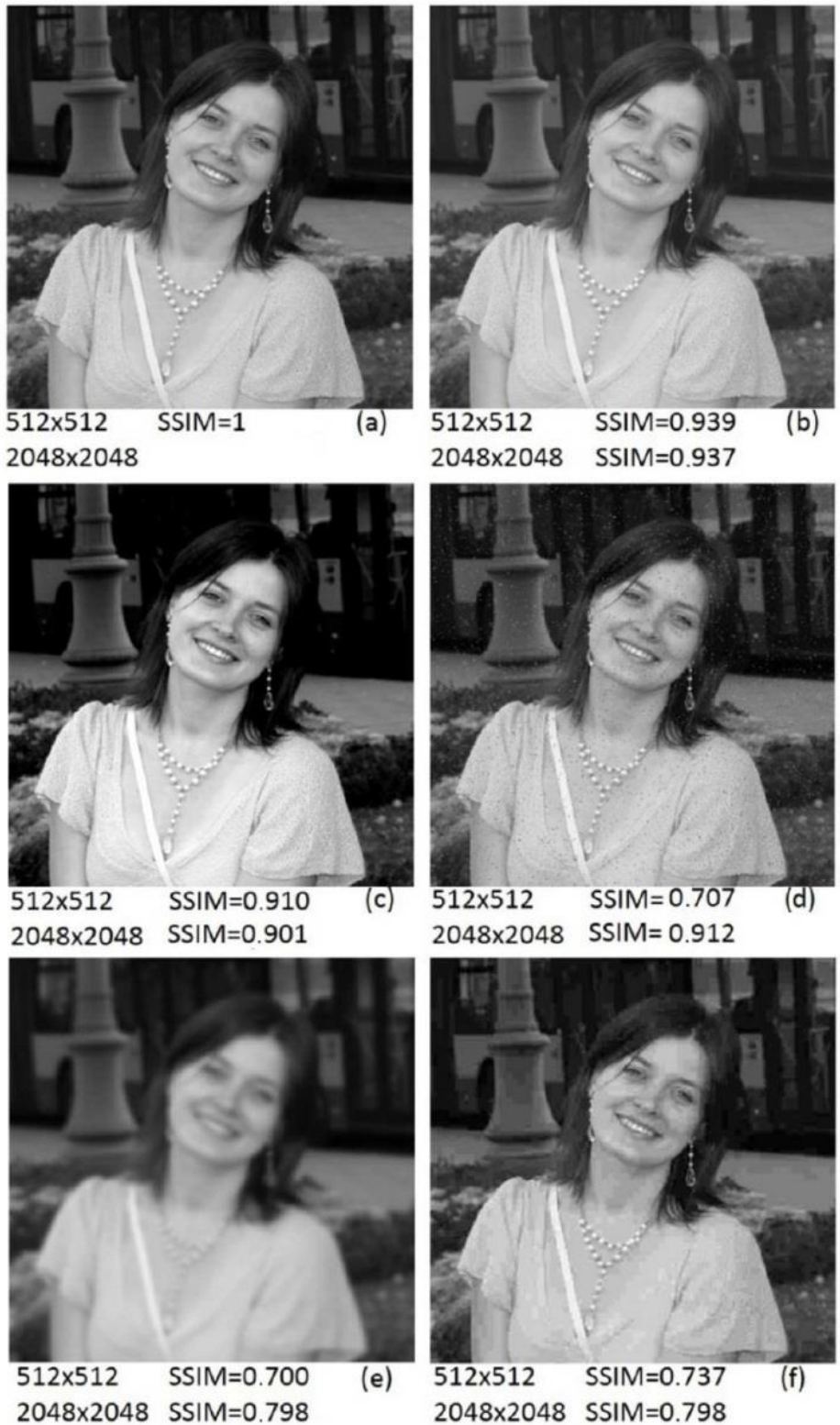

Fig. 3. The SSIM index values of distorted images, obtained by Algorithm 2.

The image downsampling can be implemented by various ways of interpolation. The main interpolation methods used for image downsampling are reviewed by Meijering in (Meijering, 2002), and the most popular methods are as follows: 
- Nearest-neighbour interpolation, where the output pixel is assigned to the value of the pixel the point of which falls within, and no other pixels are considered.

- Linear interpolation, where the output pixel value is a weighted average of pixels in the nearest 2-by-2 neighbourhood.

- Cubic interpolation, where the output pixel value is a weighted average of pixels in the nearest 4-by-4 neighbourhood.
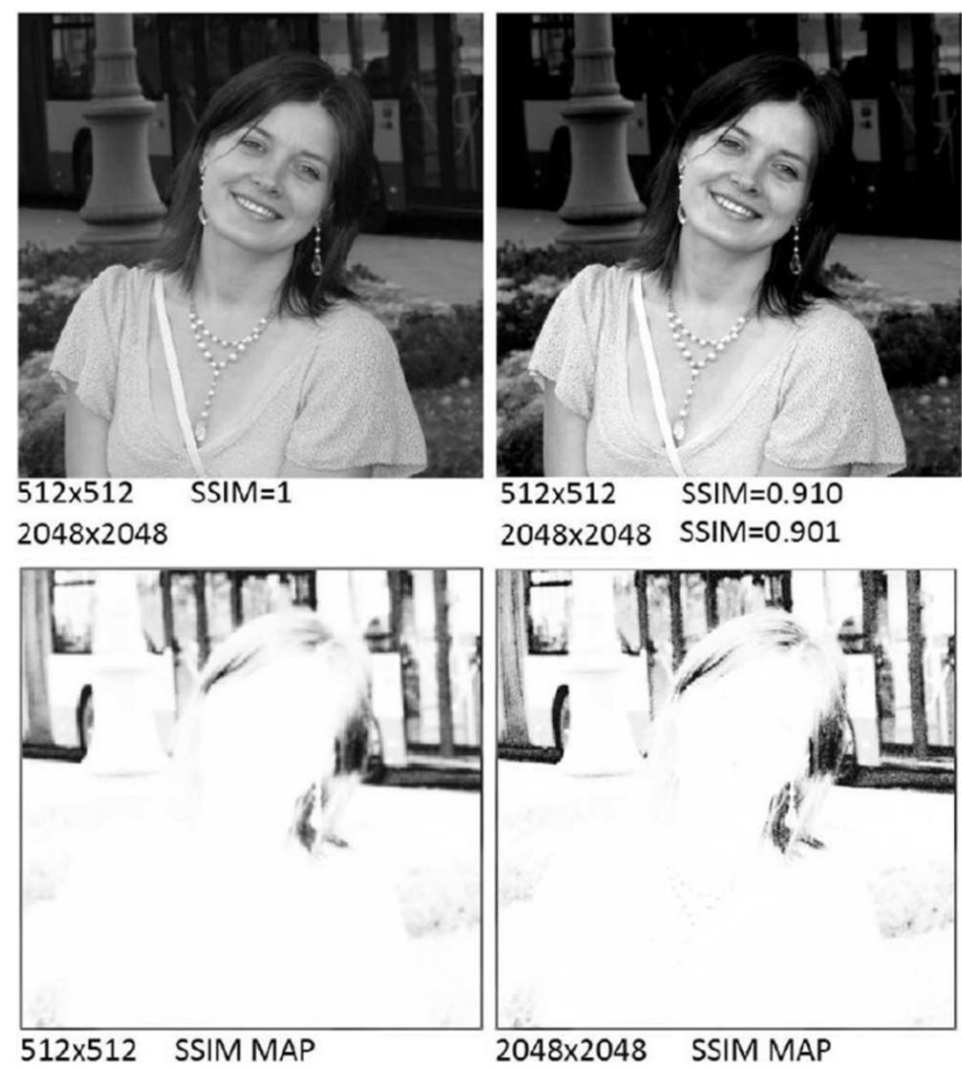

Fig. 4. The image distorted by increasing the contrast and the SSIM maps of the different size images, obtained by Algorithm 2.

.In this paper, in order to find the most suitable interpolation method for image dowsampling, the experimental investigations have been performed. The averages of the SSIM index values for the selected set of 30 images are presented in TABLE 4.

We see that the differences between the SSIM index values obtained for the original and distorted images are higher, when the nearest-neighbour interpolation is used. Therefore, we propose to use this kind of interpolation for image downsampling when small distortions of high-resolution images are investigated (we call it Algorithm 3). 

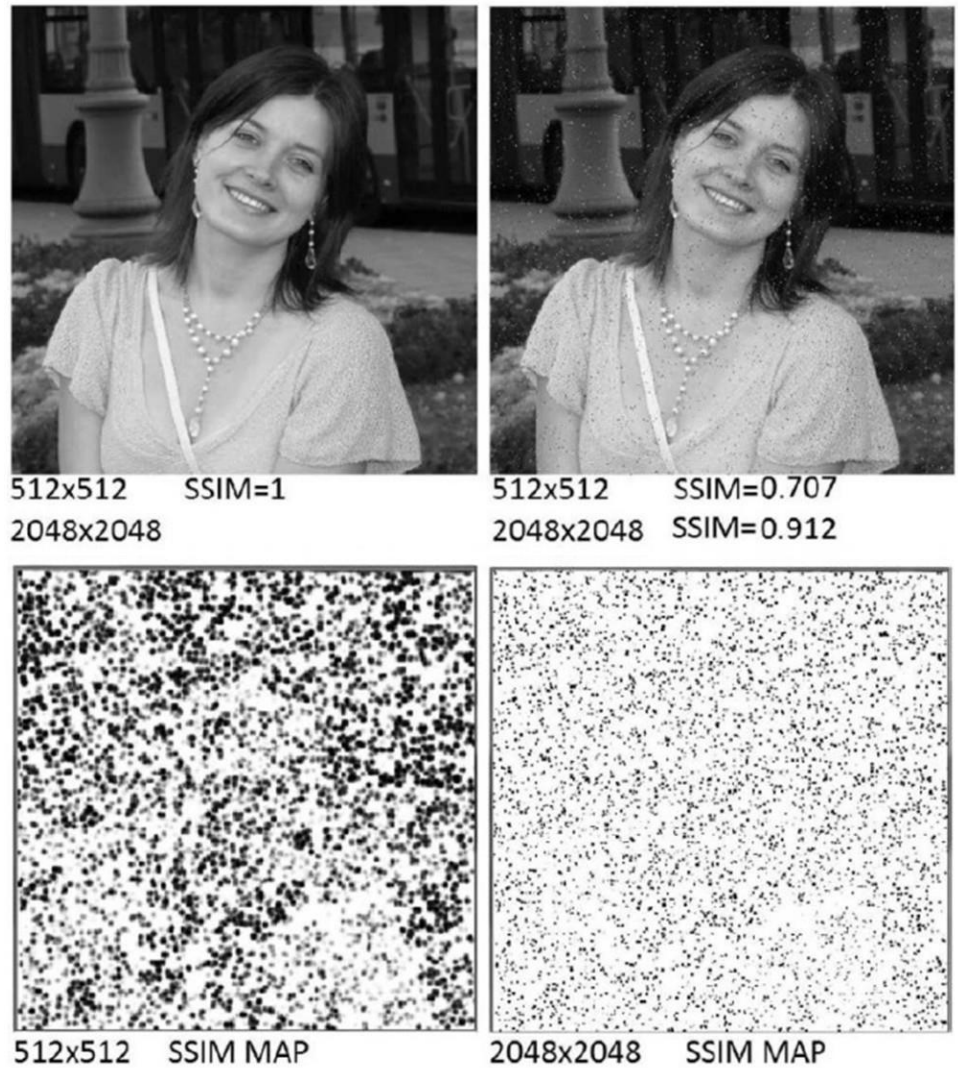

Fig. 5. The image distorted by impulsive noise and the SSIM maps of the different size images, obtained by Algorithm 2.

Table 4. The SSIM index values obtained using different interpolations

\begin{tabular}{|l|c|c|}
\hline Compression level & $\begin{array}{c}\text { Nearest- } \\
\text { neighbour }\end{array}$ & Linear \\
\hline Original & 1 & 1 \\
\hline JPEG 85 & 0.9845 & 0.9984 \\
\hline JPEG 70 & 0.9768 & 0.9982 \\
\hline JPEG 50 & 0.9672 & 0.9976 \\
\hline
\end{tabular}

Despite the fact that the type of downsampling is the only difference from the other investigated algorithms (Algorithm 1 and Algorithm 2), this difference considerably impacts the final SSIM index value (in the cases, where the size of an image is larger than $256 \times 256$ ).

The averages of the SSIM index values for a set of high-resolution 30 images, obtained by the three analysed algorithms, are presented in Table 5. 


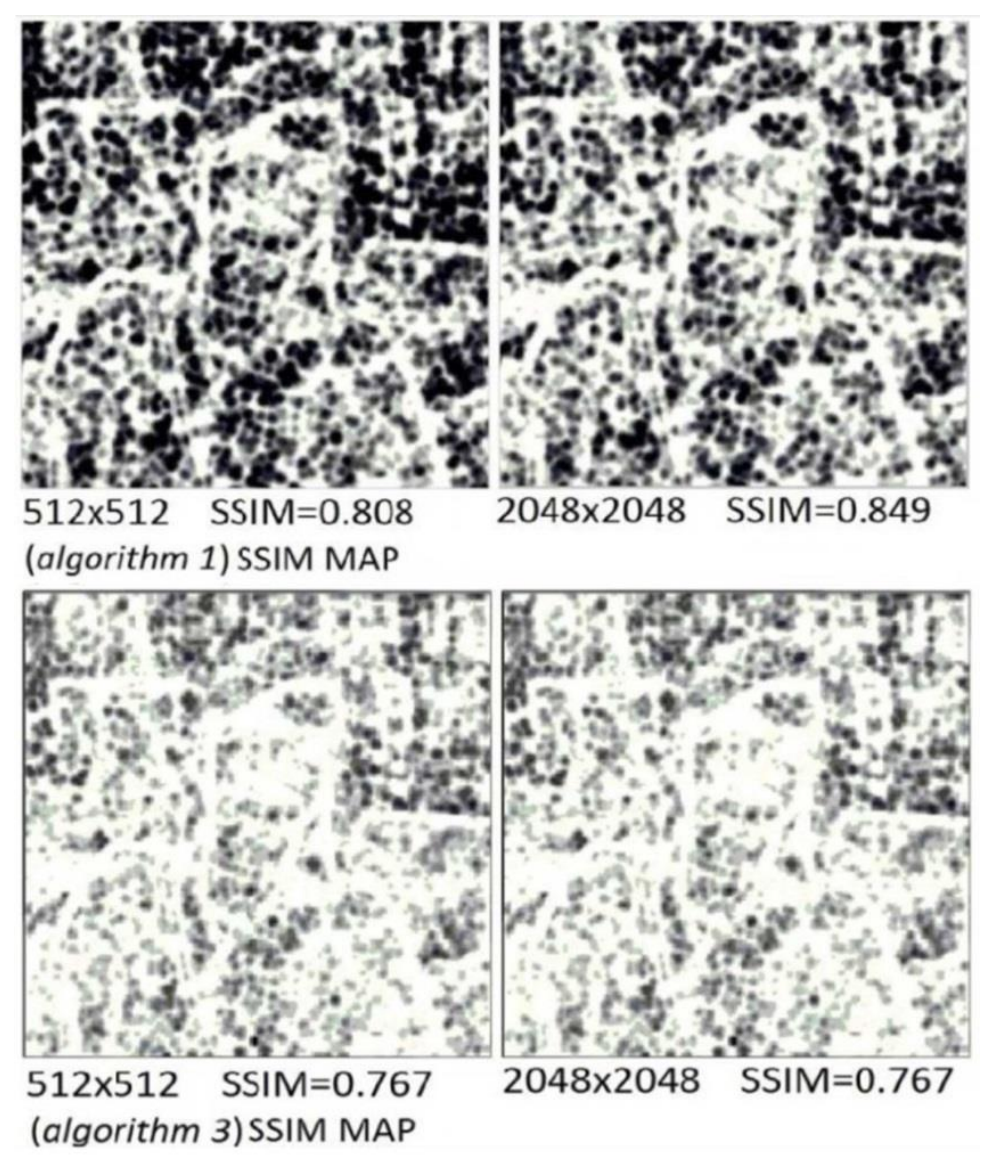

Fig. 6. The SSIM maps of different size images distorted by impulsive noise, obtained by Algorithm 1 and Algorithm 3.

Table 5. The SSIM index and inverse index values obtained by the algorithms analysed

\begin{tabular}{|l|c|c|c|}
\hline Compression level & $\begin{array}{c}\text { SSIM } \\
\text { (ISSIM) } \\
\text { Algorithm 1 }\end{array}$ & $\begin{array}{c}\text { SSIM } \\
\text { (ISSIM) } \\
\text { Algorithm 2 }\end{array}$ & $\begin{array}{c}\text { SSIM } \\
\text { (ISSIM) } \\
\text { Algorithm 3 }\end{array}$ \\
\hline Original & $1(0)$ & $1(0)$ & $1(0)$ \\
\hline JPEG 85 & $0.9999(0.01)$ & $0.9765(2.35)$ & $0.9845(1.5)$ \\
\hline JPEG 70 & $0.9998(0.02)$ & $0.9661(3.39)$ & $0.9768(2.32)$ \\
\hline JPEG 50 & $0.9985(0.15)$ & $0.9558(4.42)$ & $0.9672(3.28)$ \\
\hline
\end{tabular}

By way of illustration, we present the SSIM maps of different size of images distorted by impulsive noise, obtained by Algorithm 1 and Algorithm 3, in Fig. 6. We see that in both cases the maps are similar, thus the size of an image does not influence the values 
of the SSIM index when using both algorithms.

Algorithm 3 reflects the differences very well (TABLE 5) contrariwise to Algorithm 1. Experimental investigations of calculation speed of the SSIM index value are also performed using the proposed Algorithm 3, but only slight differences in speed compared with the original algorithm (Algorithm 1) were obtained.

For clarity of the interpretation of the obtained results, we propose to use an inverse index of similarity (ISSIM) instead of the SSIM index. It is calculated by the formula: $I S S I M=(1-S S I M) \times 100$. Hence, the higher the quality of the image, the closer to 0 the ISSIM index value is (Table 5).

\section{Conclusions}

In this paper, we have investigated the SSIM index method when assessing the quality of high-resolution images with small distortions after compression. The experimental investigation has shown that it is necessary to use image data downsampling in the algorithm for calculating the SSIM index value, otherwise, the SSIM index values are very dependent on the sizes of images, especially if their structure was changed. Moreover, it has been determined that execution of the algorithm without data downsampling is slower, and it takes much longer when the sizes of the images are large.

After the experimental investigation, where high-resolution images were processed by different variants of the algorithms for calculating the SSIM index value, we have proposed the new algorithm, where image data downsampling in the SSIM index method is implemented by the nearest-neighbour interpolation. In this case, the obtained SSIM index values better reflect the differences between the original and distorted images. The proposed algorithm will enable us to properly evaluate the quality of huge highresolution image sets automatically if distortions after applying compression algorithms are hardly noticeable.

Moreover, for clarity, we have proposed to use the inverse index of similarity (ISSIM) instead of the SSIM index for investigating the high-resolution images.

\section{References}

Brunet, D., Vrscay E. R., Wang, Z. (2012). On the mathematical properties of the structural similarity index, IEEE Transactions on Image Processing, vol. 21, no. 4, p. 1488-1499.

Chandler, D. M. (2013). Seven challenges in image quality assessment: past, present, and future research, ISRN Signal Processing, p. 53 pages.

Fernando, A., Worrall S. T., Ekmekciodlu, E. (2013). 3DTV: processing and transmission of 3D video signals, John Wiley \& Sons.

Gao, Y., Rehman A., Wang, Z. (2011). CW-SSIM based image classification, 18th International Conference on Image Processing (ICIP)., IEEE, pp. 1249-1252.

George, A. G. and Prabavathy, A. K. (2014). A survey on different approaches used in image quality assessment, International Journal of Computer Science and Network security (IJCSNS)., vol. 14, no. 2, pp. 78. 
Hore, A. and Ziou, D. (2010). Image quality metrics, PSNR vs. SSIM, 20th International Conference on Pattern Recognition (ICPR)., IEEE, pp. 2366-2369.

Huang, Y.H., Ou, T.-S., Su, P.Y., Chen, H. H. (2010). Perceptual rate-distortion optimization using structural similarity index as quality metric, IEEE Transactions on Circuits and Systems for Video Technology, vol. 20, no. 11, pp. 1614-1624.

Yang, C., Gao, W., Po, L. (2008). Discrete wavelet transform-based structural similarity for image quality assessment, 15th IEEE International Conference on Image Processing (ICIP)., IEEE, p. 377-380.

Ji, G., Ni, X., Bae, H. (2008). A full-reference image quality assessment algorithm based on haar wavelet transform, International Conference on Computer Science and Software Engineering, IEEE, vol. 1, p. 791-794.

Joy K., and Sarma, E. G. (2014). Recent developments in image quality assessment algorithms, A review Journal of Theoretical and Applied Information Technology, vol. 65, no. 10, pp. 192-201.

Meijering, E. (2002). A chronology of interpolation: from ancient astronomy to modern signal and image processing, Proceedings of the IEEE, vol. 90, no. 3, pp. 319342.

Piella, G., Cwi, A. N., Heijmans, H. (2003). A new quality metric for image fusion, International Conference on Image Processing (ICIP)., IEEE, pp. III - 173-6 vol.2.

Ponomarenko, N., Battisti, F., Egiazarian, K., Astola, J., Lukin, V. (2009b). Metrics performance comparison for color image database, Fourth international workshop on video processing and quality metrics for consumer electronics, vol. 27.

Ponomarenko, N., Ieremeiev, O., Lukin V., Egiazarian, K. (2013). Color image database TID2013: Peculiarities and preliminary results, (EUVIP),, 4th European Workshop on Visual Information Processing, pp. 106-111.

Ponomarenko, N., Lukin, V., Zelensky, A., Egiazarian, K., Carli, M., Battisti, F. (2009a). TID2008 - A database for evaluation of full-reference visual quality assessment metrics, Advances of Modern Radioelectronics, vol. 10, no. 4, pp. 3045.

Rehman, A., Rostami, M., Wang, Z., Brunet D., Vrscay, E. R. (2012). SSIM - inspanded image restoration using sparse representation, EURASIP Journal on Advances in Signal Processing, vol. 1, pp. 1-12.

Russ, J. C. (2011). The image processing handbook, CRC press.

Salomon, D. (2013). A guide to data compression methods, Springer Science \& Business Media.

Skodras, A., Christopoulos C., Ebrahimi, T. (2001). The JPEG2000 still image compression standard, Signal Processing Magazine, IEEE, vol. 18, no. 5, pp. 3658.

Thung, K. and Raveendran, P. (2009). A survey of image quality measures. International Conference for Technical Postgraduates, (TECHPOS, 2009). IEEE, pp. 1-4.

Wallace, G. K. (1992). The JPEG still picture compression standard, IEEE Transactions on Consumer Electronics, vol. 38, no. 1, pp. 18-34.

Wang, Z. and Bovik, A. C. (2009). Mean squared error: love it or leave it? A new look at signal fidelity measures, Signal Processing Magazine, IEEE, vol. 26, no. 1, pp. 98-117. 
Wang, Z., Bovik, A. C., Sheikh, H. R., Simoncelli, E. P. (2004a). Image quality assessment: from error visibility to structural similarity, IEEE Transactions on Image Processing, vol. 13, no. 4, pp. 600-612.

Wang, Z., Simoncelli E. P., Bovik, A. C. (2004b). Multiscale structural similarity for image quality assessment, Conference Record of the Thirty-Seventh Asilomar Conference on Signals, Systems and Computers, IEEE, vol. 2, p. 1398-1402.

WEB (a). GNU Image Manipulation Programm. http://http://www.gimp.org/.

WEB (b). Independent JPEG Group. http://www.ijg.org/.

WEB (c). Wang's MATLAB function. SSIM index with automatic downsampling, version 1.0 copyright(c). https://ece.uwaterloo.ca/ z70wang/research/ssim/.

Winkler, S. (2012). Analysis of public image and video databases for quality assessment, IEEE Journal of Selected Topics in Signal Processing, vol. 6, no. 6, pp. 616-625.

Received April 11, 2017, revised June 7, 2017, accepted June 14, 2017 